\title{
Fabrication of high quality strained SiGe on Si substrate by RPCVD
}

\author{
XUE Zhong Ying ${ }^{1 *}$, CHEN Da $^{3}$, LIU LinJie ${ }^{1,2}$, JIANG HaiTao $^{1}$, BIAN JianTao ${ }^{1}$, WEI Xing ${ }^{1}$, \\ DI ZengFeng ${ }^{1}$, ZHANG Miao ${ }^{1} \&$ WANG Xi ${ }^{1}$ \\ ${ }^{1}$ State Key Laboratory of Functional Materials for Informatics, Shanghai Institute of Microsystem and Information Technology, Chinese Academy \\ of Sciences, Shanghai 200050, China; \\ ${ }^{2}$ Graduate University of Chinese Academy of Sciences, Beijing 100049, China; \\ ${ }^{3}$ Department of Physics, Lanzhou University, Lanzhou 730000, China
}

Received October 26, 2011; accepted December 30, 2011; published online March 23, 2012

In this study, the growth kinetics of $\mathrm{SiGe}$ in a reduced pressure chemical vapor deposition system using dichlorosilane $\left(\mathrm{SiH}_{2} \mathrm{Cl}_{2}\right)$ and germane $\left(\mathrm{GeH}_{4}\right)$ as the $\mathrm{Si}$ and $\mathrm{Ge}$ precursors were investigated. The $\mathrm{SiGe}$ growth rate and $\mathrm{Ge}$ content were found to depend on the deposition temperature, $\mathrm{GeH}_{4}$ flow and reactor chamber pressure. The SiGe growth rate escalates with increasing deposition temperature, while the Ge content is reduced. The $\mathrm{SiGe}$ growth rate accelerates with increasing $\mathrm{GeH}_{4}$ flow, while the Ge content increases more slowly. According to the experimental data, a new relationship between Ge content $(x)$ and $\mathrm{F}\left(\mathrm{GeH}_{4}\right) / \mathrm{F}\left(\mathrm{SiH}_{2} \mathrm{Cl}_{2}\right)$ mass flow ratio is deduced: $x^{2.5} /(1-x)=n \mathrm{~F}\left(\mathrm{GeH}_{4}\right) / \mathrm{F}\left(\mathrm{SiH}_{2} \mathrm{Cl}_{2}\right)$. The SiGe growth rate and Ge content improve with increasing reactor chamber pressure. By selecting proper precursor flows and reactor pressure, SiGe films with the same Ge content can be fabricated at various temperatures. However, the quality of the SiGe crystals is clearly dependent on the deposition temperature. At lower deposition temperature, higher crystalline quality is achieved. Because the growth rate dramatically drops with lower temperatures, the optimum growth temperature must be a compromise between the crystalline quality and the growth rate. X-ray diffraction, Raman scattering spectroscopy and atomic force microscopy results indicate that $650^{\circ} \mathrm{C}$ is the optimum temperature for fabrication of $\mathrm{Si}_{0.75} \mathrm{Ge}_{0.25}$ film.

SiGe, epitaxial growth, growth rate, Ge content

Citation: Xue Z Y, Chen D, Liu L J, et al. Fabrication of high quality strained SiGe on Si substrate by RPCVD. Chin Sci Bull, 2012, 57: 1862-1867, doi: $10.1007 / \mathrm{s} 11434-012-5020-7$

Over the years, there has been keen interest in fabricating high performance metal oxide semiconductor field effect transistors (MOSFETs) with strained $\mathrm{Si} / \mathrm{SiGe}$ channels [1-3], as the carrier mobility in the strained layer is notably enhanced compared with that of Si MOSFETs. Defects in the strained layer, however, degrade carrier mobility and increase the leakage current. Thus, the crystal quality of the strained layer is an important factor in improving the performance of the MOSFETs. High quality strained $\mathrm{SiGe} / \mathrm{Si}$ heterostructures can be fabricated by molecular beam epitaxy (MBE) [4,5] or by chemical vapor deposition (CVD) $[6,7]$. Compared with MBE, CVD technology is more satis-

*Corresponding author (email: simsnow@mail.sim.ac.cn) factory for industrial semiconductor production.

Several CVD technologies, such as atmospheric pressure CVD (APCVD) [8,9], ultra-high vacuum CVD [10], and reduced pressure CVD (RPCVD) [11,12], have been applied to fabricate $\mathrm{SiGe} / \mathrm{Si}$ heterostructures. Among them, RPCVD is the most convenient method to grow high quality $\mathrm{SiGe}$ layers at reasonable growth rates in the semiconductor industry [13-15].

During SiGe deposition in an RPCVD chamber, all the growth parameters such as deposition temperature, Ge/Si precursor flow ratio and reactor chamber pressure can affect the SiGe growth rate and Ge content; thus SiGe layers with the same Ge content can probably be grown with different combinations of growth parameters. However, their crystal- 
line quality may differ distinctly. In fact, the growth parameters have a crucial role in reducing the defect density. Thus, research on how the $\mathrm{SiGe}$ growth rate and Ge content depend on growth parameters serves as the basis for selecting appropriate parameters to fabricate high quality strained SiGe.

\section{Experimental}

All the strained SiGe samples were grown on p-type $\mathrm{Si}(100)$ substrates $(10-20 \Omega \mathrm{cm})$ in an Epsilon 2000 ASM CVD system, which is a horizontal, single wafer, load-locked reactor. Before SiGe films were deposited, Si substrates were cleaned in the standard RCA-1 and RCA-2 solutions and rinsed in deionized water. After the $\mathrm{Si}$ substrate was loaded into the chamber, an in-situ bake in $\mathrm{H}_{2}$ at $1060^{\circ} \mathrm{C}$ was performed to remove any remaining oxide. Keeping the reactor chamber pressure at 1.33-10.64 $\mathrm{kPa}$, epitaxial growth was carried out at $550-900^{\circ} \mathrm{C}$ using dichlorosilane $\left(\mathrm{SiH}_{2} \mathrm{Cl}_{2}\right)$ as the $\mathrm{Si}$ precursor and $10 \%$ germane $\left(\mathrm{GeH}_{4}\right)$ in $\mathrm{H}_{2}$ as the Ge precursor. During deposition, the flow of $\mathrm{H}_{2}$ carrier gas was set at a fixed value of $20 \mathrm{slms}$, and the flow of $\mathrm{SiH}_{2} \mathrm{Cl}_{2}$ at a fixed value of $75 \mathrm{sccms}$. The structural properties of the $\mathrm{SiGe}$ samples were investigated by high-resolution X-ray diffraction (XRD), Raman scattering spectroscopy and atomic force microscopy (AFM).

\section{Results and discussion}

\subsection{The dependence of SiGe growth rate and Ge con- tent on deposition temperature}

After growing pseudomorphic $\mathrm{SiGe/Si}$ heterostructures using particular deposition parameters, the thickness and $\mathrm{Ge}$ content of the SiGe layers were determined by X-ray diffraction. Since the XRD peak of relaxed SiGe moves towards higher diffraction angles with increasing extent of relaxation, the exact Ge content can only be extracted from the XRD pattern once the relaxation degree of the $\mathrm{SiGe}$ sample has been determined. Figure 1 shows the high resolution XRD patterns of $\mathrm{SiGe}$ samples fabricated at varied deposition temperatures with fixed precursor flow and reactor chamber pressure. The presence of thickness interference peaks in these XRD spectra indicates that all these SiGe samples are fully strained, i.e., their relaxation degrees are all $0 \%$. As the deposition temperature declines, the SiGe peak deviates away from the Si substrate peak, which means that the Ge concentration has increased. However, when the temperature drops to $550^{\circ} \mathrm{C}$, the $\mathrm{SiGe}$ peak disappears from the XRD pattern (not shown in Figure 1), because it is difficult to decompose $\mathrm{SiH}_{2} \mathrm{Cl}_{2}$ at this temperature. For those $\mathrm{SiGe}$ samples fabricated at $600-900^{\circ} \mathrm{C}$, the $\mathrm{SiGe}$ layer thickness and the Ge concentration were extracted based on the Takagi-Taupin dynamical scattering theory [16].

Table 1 lists the Ge content and growth rate (thickness/

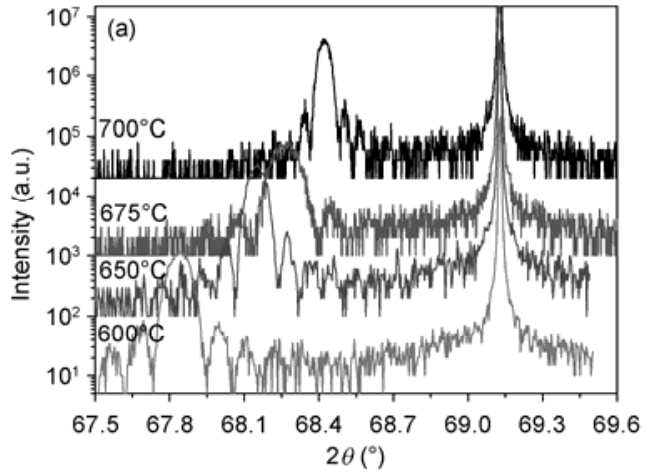

Figure 1 XRD patterns of SiGe samples deposited at different temperatures with fixed precursor flow and reactor chamber pressure.

Table 1 Dependence of SiGe growth rate (GR) and Ge content on deposition temperature at fixed reactor pressure and precursor flows

\begin{tabular}{|c|c|c|c|c|c|}
\hline $\begin{array}{c}\text { Temperature } \\
\left({ }^{\circ} \mathrm{C}\right)\end{array}$ & $\begin{array}{l}\text { SiGe GR } \\
(\mathrm{nm} / \mathrm{min})\end{array}$ & $\mathrm{Ge}(\%)$ & $\begin{array}{c}\text { Ge GR } \\
(\mathrm{nm} / \mathrm{min})\end{array}$ & $\begin{array}{c}\text { Si GR } \\
(\mathrm{nm} / \mathrm{min})\end{array}$ & $\begin{array}{c}\text { Ge:Si } \\
\text { composition ratio }\end{array}$ \\
\hline 600 & 3.5 & 24.4 & 0.85 & 2.65 & 0.32 \\
\hline 675 & 10 & 17.0 & 1.7 & 8.3 & 0.2 \\
\hline 700 & 20 & 14.4 & 2.88 & 17.12 & 0.17 \\
\hline 725 & 40 & 12.4 & 4.96 & 35.04 & 0.14 \\
\hline 750 & 51.7 & 10.8 & 5.58 & 46.12 & 0.12 \\
\hline 800 & 83 & 9 & 7.4 & 75.5 & 0.10 \\
\hline 900 & 200 & 5.5 & 11 & 189 & 0.06 \\
\hline
\end{tabular}


deposition time) determined from the XRD patterns of Figure 1. As shown in Table 1 , the $\mathrm{SiGe}$ growth rate rises sharply from 3.5 to $200 \mathrm{~nm} / \mathrm{min}$ when the temperature increases from 600 to $900^{\circ} \mathrm{C}$, but the Ge content decreases from $24.4 \%$ to $5.5 \%$ over this temperature range. The SiGe samples fabricated at 800 and $900^{\circ} \mathrm{C}$ are particularly noteworthy. Although the deposition temperature is rather high, the SiGe layers remain fully strained (as confirmed from Raman spectra, which are not shown here) because of the low Ge content. Based on the Ge content and $\mathrm{SiGe}$ growth rate, the respective growth rates of $\mathrm{Si}$ and $\mathrm{Ge}$ in the $\mathrm{SiGe}$ layer were calculated and plotted in Figure 2 as a function of the inverse absolute temperature. It can easily be seen from this Arrhenius plot that the $\mathrm{Si}$ and $\mathrm{Ge}$ growth rates are thermally activated. The calculated activation energies for $\mathrm{Si}$ and $\mathrm{Ge}$ are 1.79 and $1.27 \mathrm{eV}$, respectively. According to Figure 2, the relationships between the $\mathrm{Si}$ growth rate $\left(R_{\mathrm{Si}}\right)$, Ge growth rate $\left(R_{\mathrm{Ge}}\right)$ and absolute temperature $(T)$ can be described as

$$
\begin{gathered}
R_{\mathrm{Si}} \approx C_{\mathrm{Si}} \mathrm{e}^{-\frac{E_{\mathrm{A}(\mathrm{Si})}}{k T}}, \\
R_{\mathrm{Ge}} \approx C_{\mathrm{Ge}} \mathrm{e}^{-\frac{E_{\mathrm{A}(\mathrm{Ge})}}{k T}},
\end{gathered}
$$

where $C_{\mathrm{Si}}$ and $C_{\mathrm{Ge}}$ are constants, and $E_{\mathrm{A}(\mathrm{Si})}$ and $E_{\mathrm{A}(\mathrm{Ge})}$ are activation energies for $\mathrm{Si}$ and $\mathrm{Ge}$ growth, respectively. The relationship between the Ge content $(x)$ of the SiGe layer and the deposition temperature can be deduced from eqs. (1) and (2):

$$
\frac{x}{1-x}=\frac{R_{\mathrm{Ge}}}{R_{\mathrm{Si}}} \approx C_{1} \mathrm{e}^{\frac{E_{\mathrm{A}(\mathrm{Si})}-E_{\mathrm{A}(\mathrm{Ge})}}{k T}} \quad(873 \mathrm{~K} \leqslant T \leqslant 1173 \mathrm{~K}),
$$

where $E_{\mathrm{A}(\mathrm{Si})}-E_{\mathrm{A}(\mathrm{Ge})}$ is equal to $0.52 \mathrm{eV}$, and $C_{1}$ is a constant. Eq. (3) can be rewritten as

$$
\ln \left(\frac{x}{1-x}\right) \approx \ln C_{1}+C_{2} \frac{1}{T} \quad(873 \mathrm{~K} \leqslant T \leqslant 1173 \mathrm{~K}),
$$

where $C_{2}$ is a constant. The inset of Figure 2 shows the relationship between the actual Ge:Si content ratio and the inverse absolute temperature; the graph agrees with eq. (4).

\subsection{Dependence of $\mathrm{SiGe}$ growth rate and Ge content on $\mathrm{GeH}_{4}$ flow}

As shown in Figure 3(a), the SiGe growth rate accelerates with increasing $\mathrm{GeH}_{4}$ flow; the Ge content, however, climbs more slowly. Since the steady state concentration of free Ge sites is much higher than that of free Si sites during deposition of SiGe by RPCVD, equilibrium is achieved by transferring the $\mathrm{H}$ adatoms from $\mathrm{Si}$ to $\mathrm{Ge}$. As the energy barrier for $\mathrm{H}_{2}$ desorption from the Ge surface is lower than that for $\mathrm{H}_{2}$ desorption from the $\mathrm{Si}$ surface, the growth rate enhancement with increasing $\mathrm{GeH}_{4}$ flow can be explained by the lowering of energy barriers for hydrogen desorption

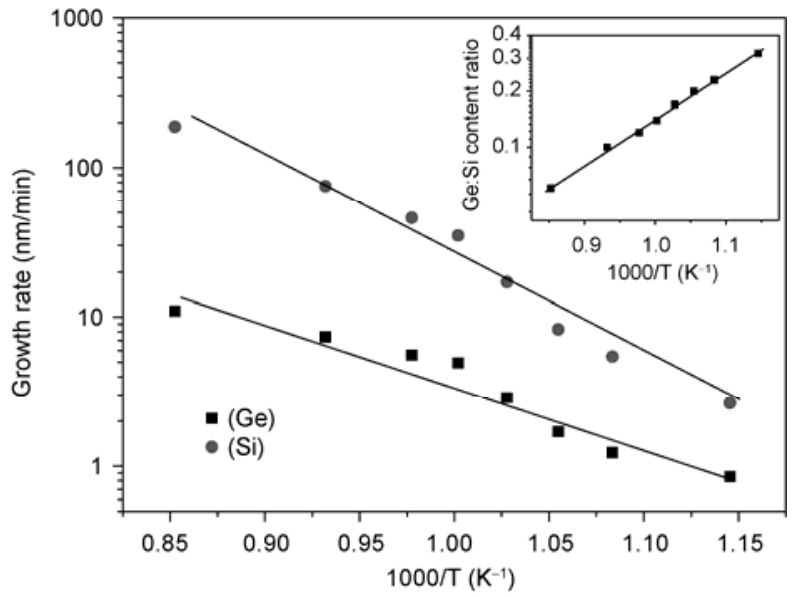

Figure 2 The growth rates of $\mathrm{Si}$ and $\mathrm{Ge}$ in the SiGe layer as a function of the inverse absolute temperature. The inset shows the dependence of Ge:Si content ratio on deposition temperature.

[17-20]. According to the literature [13,21], the ratio of $x^{2} /(1-x): \mathrm{F}\left(\mathrm{GeH}_{4}\right) / \mathrm{F}\left(\mathrm{SiH}_{2} \mathrm{Cl}_{2}\right)$ should be a constant if the flows of $\mathrm{H}_{2}$ carrier gas, $\mathrm{HCl}$ and $\mathrm{SiH}_{2} \mathrm{Cl}_{2}$ are all fixed. Our experimental data, however, indicate that a constant ratio is only obtained when the exponent of Ge content $(x)$ is modified to give $x^{2.5} /(1-x): \mathrm{F}\left(\mathrm{GeH}_{4}\right) / \mathrm{F}\left(\mathrm{SiH}_{2} \mathrm{Cl}_{2}\right)$, as shown in Figure 3(b). When $\mathrm{F}(\mathrm{HCl})=0$ and $\mathrm{F}\left(\mathrm{SiH}_{2} \mathrm{Cl}_{2}\right) / \mathrm{F}\left(\mathrm{H}_{2}\right)=$ 0.00375 , the ratio $x^{2.5} /(1-x): \mathrm{F}\left(\mathrm{GeH}_{4}\right) / \mathrm{F}\left(\mathrm{SiH}_{2} \mathrm{Cl}_{2}\right)$ is 0.7 . Compared with the reported result [21], our revised relationship implies that a higher Ge content could be obtained if SiGe films were deposited with the same parameters. Considering the effect of temperature on SiGe epitaxy, a reasonable explanation for this difference is that our actual epitaxial temperature is lower than the nominal temperature, because the thermocouple is not in contact with the Si substrate when SiGe is deposited.

\subsection{Dependence of SiGe growth rate and Ge content on reactor chamber pressure}

The SiGe growth rate and Ge content as a function of reactor chamber pressure are plotted in Figure 4. The striking improvement in the $\mathrm{SiGe}$ growth rate is because more $\mathrm{Si}$ and Ge precursors are supplied with increasing reactor chamber pressure. A high reactor chamber pressure produces substantial quantities of Cl-based molecules, which, in turn, suppresses the decomposition of $\mathrm{SiH}_{2} \mathrm{Cl}_{2}$. As a result, the Ge content increases with the reactor chamber pressure.

\subsection{The fabrication of high quality strained SiGe}

The above analysis suggests that although SiGe layers with the same Ge content can be fabricated at different temperatures, the crystalline quality is, to a great extent, affected by the deposition temperature. Figure 5 shows the XRD patterns of two SiGe samples fabricated at $650^{\circ} \mathrm{C}$ (sample A) 

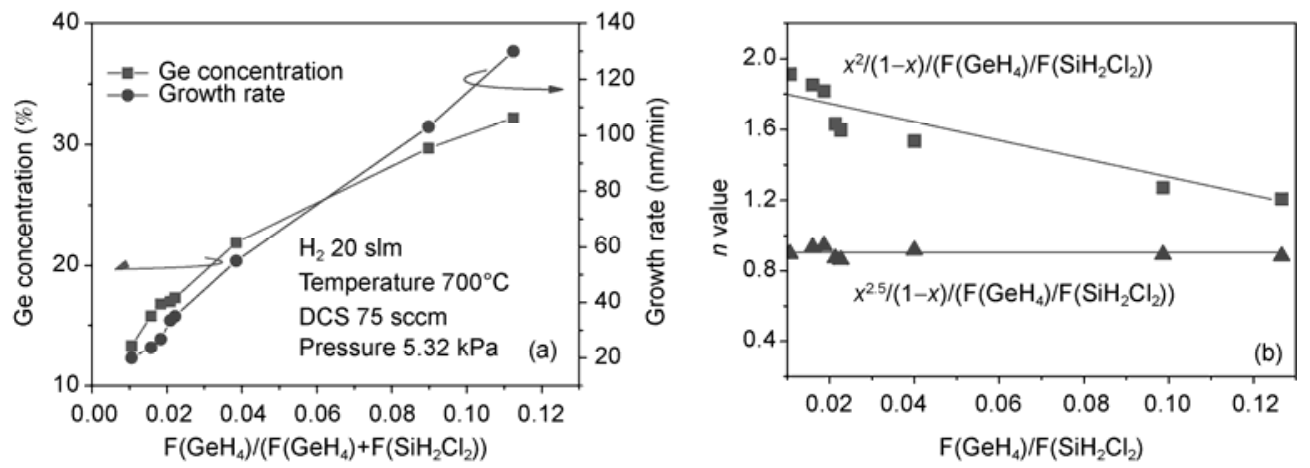

Figure 3 (a) The SiGe growth rate speeds up notably with increasing $\mathrm{GeH}_{4}$ flow. However, the Ge concentration climbs more slowly; (b) the different relationships between $\mathrm{Ge}$ content $(x)$ and $\mathrm{F}\left(\mathrm{GeH}_{4}\right) / \mathrm{F}\left(\mathrm{SiH}_{2} \mathrm{Cl}_{2}\right)$ mass flow ratio.

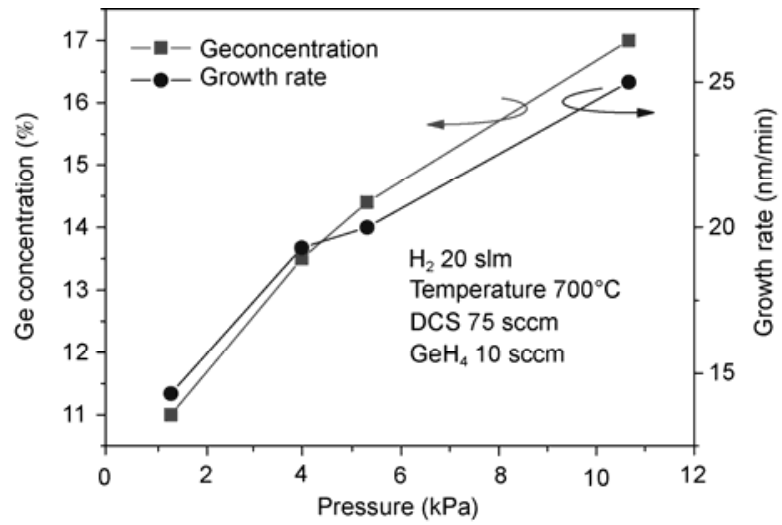

Figure 4 The SiGe growth rate and Ge concentration increase significantly with increasing reactor chamber pressure.

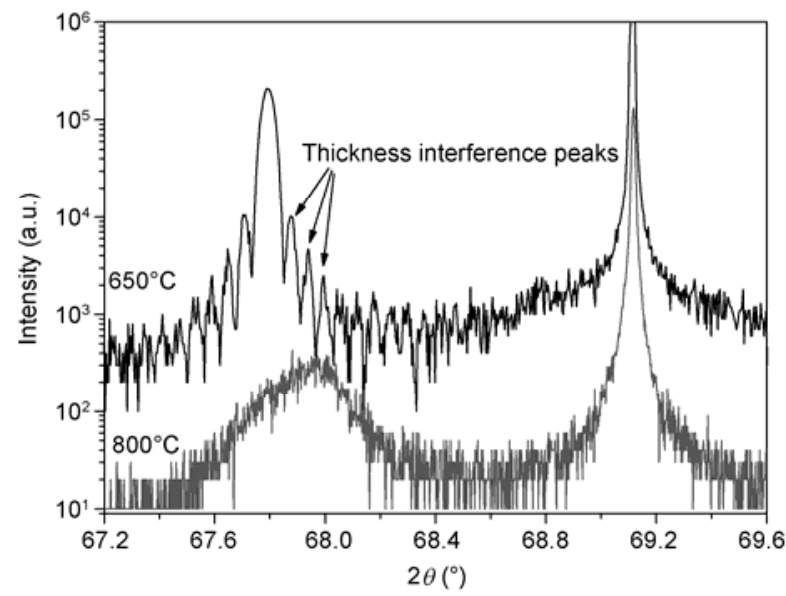

Figure 5 XRD patterns of SiGe fabricated at $650^{\circ} \mathrm{C}$ (sample A) and $800^{\circ} \mathrm{C}$ (sample B). The sharp SiGe peak and the presence of thickness interference peaks indicate the good crystalline quality of sample A. The broadened SiGe peak and the almost indiscernible thickness interference peaks demonstrate a decline in SiGe crystalline quality for sample B.

and $800^{\circ} \mathrm{C}$ (sample B). For sample A, the narrow full width at half maximum (FWHM) of the SiGe peak (only $0.1^{\circ}$ ) and the presence of thickness interference peaks indicate that the $\mathrm{SiGe}$ layer is pseudomorphic to the $\mathrm{Si}$ substrate, i.e. there is negligible dislocation density at the $\mathrm{SiGe} / \mathrm{Si}$ interface. The SiGe layer thickness and the Ge content extracted from the XRD pattern are $151 \mathrm{~nm}$ and $25.0 \%$, respectively. For sample $\mathrm{B}$, the SiGe peak not only broadens significantly, but also becomes asymmetrical. In addition, the thickness interference peaks almost disappear from the XRD pattern. All of these features are associated with plastic relaxation of compressive strain and decline of the SiGe crystalline quality. As a result, the thickness and the Ge content of sample $\mathrm{B}$ become unidentifiable using XRD. To further evaluate the Ge content and relaxation degree, the Raman spectra of samples A and B were measured at room temperature using a JOBIN YVON HR800 spectrometer with a $514 \mathrm{~nm}$ excitation wavelength, and are shown in Figure 6. The in-plane strain and Ge content can be evaluated simultaneously from the Raman shifts of the Si-Si, and Si-Ge peaks [22]. Sample A is fully strained and its Ge content is $25 \%$, which is consistent with the XRD result; sample B is partially relaxed with a relaxation of $14 \%$, though its Ge content is only $21 \%$.

As shown by the XRD and Raman results, sample B shows inferior crystalline quality although its thickness and Ge content are lower than those of sample A; this can be

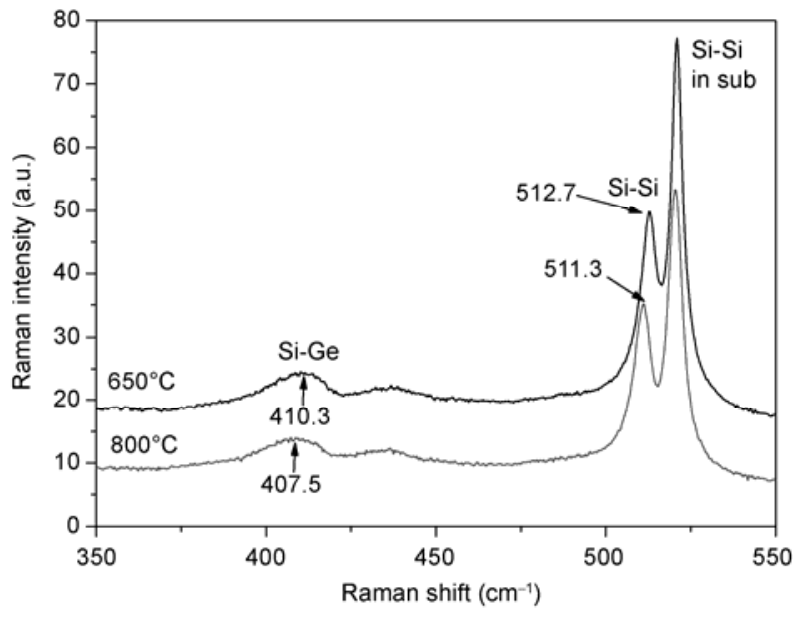

Figure 6 Raman spectra of sample A $\left(650^{\circ} \mathrm{C}\right)$ and sample $\mathrm{B}\left(800^{\circ} \mathrm{C}\right)$. 

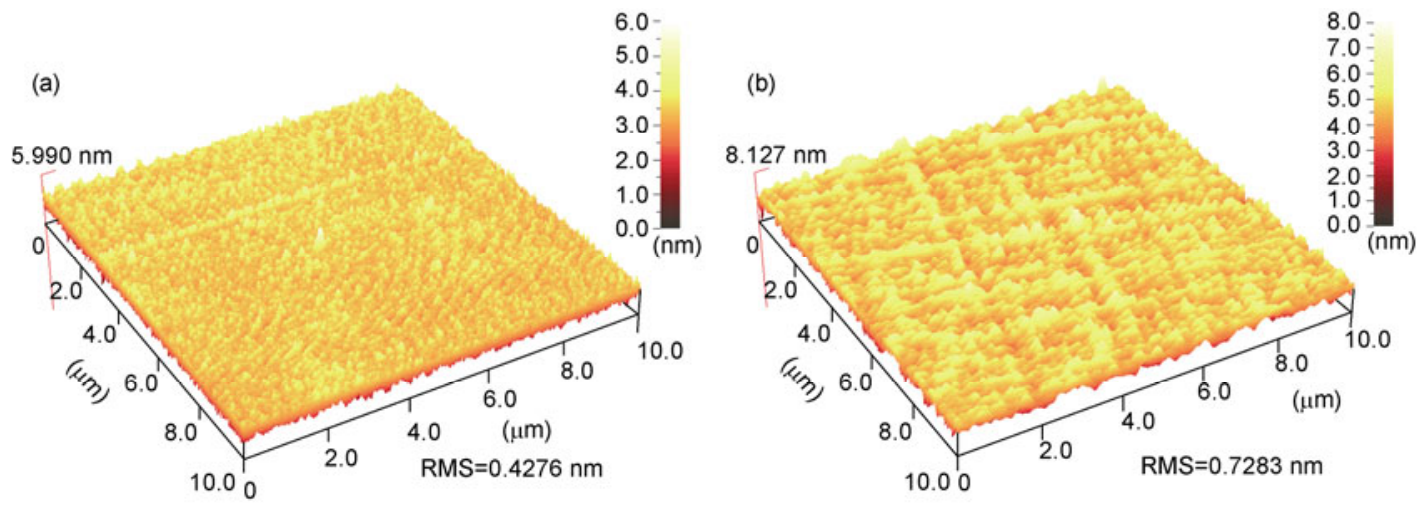

Figure 7 AFM images of samples A and B.

ascribed to its high deposition temperature. According to the suggested relaxation mechanism of a heteroepitaxial SiGe layer during growth on a Si substrate [23], the strain energy density plays a key role in the relaxation of the SiGe film. During growth of $\mathrm{SiGe}$ on a $\mathrm{Si}$ substrate, the strain energy density accumulates gradually as the SiGe film gets thicker. When the strain energy density reaches a critical value, it is relaxed by the formation of dislocations [24], resulting in the relaxation of the SiGe film and the decline of the crystalline quality. The higher the deposition temperature, the more strain energy is accumulated. Consequently, the $\mathrm{SiGe}$ layer fabricated at higher temperature begins to relax despite its thinner film thickness and lower Ge content.

Compared with sample B, sample A demonstrates not only superior crystalline quality, but also good surface morphology. Figure 7 shows the AFM images of samples A and $\mathrm{B}$. The root-mean-square (RMS) roughness of sample A in a $10 \mu \mathrm{m}$ square is only $0.43 \mathrm{~nm}$; for sample $\mathrm{B}$, the RMS roughness is $0.73 \mathrm{~nm}$. Besides the bigger RMS value, a cross-hatch pattern was observed in the AFM image of sample $\mathrm{B}$, where ridges and trenches extend in the $<110>$ directions on the surface. It has been reported in the literature that the cross-hatch is caused by the relaxation of strain fields [25], which is consistent with the Raman result of sample B.

The XRD, Raman and AFM data for samples A and B suggest that a low deposition temperature is the essential factor to grow high quality SiGe. The higher the Ge content, the lower the required deposition temperature. However, the decrease in deposition temperature is necessarily accompanied by a drop in the growth rate. The optimal growth temperature must be a compromise between the crystalline quality and growth rate.

\section{Conclusion}

The dependence of the SiGe growth rate and Ge content on deposition temperature, $\mathrm{GeH}_{4}$ flow and reactor chamber pressure was investigated. The $\mathrm{SiGe}$ growth rate escalates with increasing deposition temperature; meanwhile, the Ge content decreases, which can be explained by the Arrhenius equation. Because Ge incorporation lowers the energy barriers for hydrogen desorption, high $\mathrm{GeH}_{4}$ flow also leads to rapid $\mathrm{SiGe}$ deposition rate. Although the decomposition rates of both $\mathrm{SiH}_{2} \mathrm{Cl}_{2}$ and germane increase notably at high pressure, high pressure is more favorable for the decomposition of germane, because the presence of substantial quantities of Cl-based molecules impedes the decomposition of $\mathrm{SiH}_{2} \mathrm{Cl}_{2}$. Consequently, the rise of $\mathrm{SiGe}$ growth rate at high reactor pressure is accompanied by an increase in Ge content.

Although SiGe layers can be fabricated at different temperatures, the rapid accumulation of strain energy at high temperature lowers the crystalline quality. To fulfill the semiconductor industry requirements for both crystalline quality and growth rate, SiGe deposition temperature must be carefully optimized.

This work was supported by the National Natural Science Foundation of China (61006088), the National Basic Research Program of China (2010CB832906), and the Natural Science Foundation of Shanghai (10ZR1436100).

1 Mooney P M, Chu J O. SiGe technology: Heteroepitaxy and highspeed microelectronics. Annu Rev Mater Sci, 2000, 30: 335-362

2 Tezuka T, Nakaharai S, Moriyama Y, et al. Strained SOI/SGOI dual-channel CMOS technology based on the Ge condensation technique. Semicond Sci Tech, 2007, 22: S93-S98

3 Cheng Z, Currie M, Leitz C, et al. Electron mobility enhancement in strained-Si n-MOSFETs fabricated on SiGe-on-insulator (SGOI) substrates. IEEE Electr Device L, 2001, 22: 321-323

4 Spadafora M, Terrasi A, Mirabella S, et al. Dry oxidation of MBE-SiGe films: Rate enhancement, Ge redistribution and defect injection. Mat Sci Semicon Proc, 2005, 8: 219-224

5 Kasper E, Lyutovich K. Strain adjustment with thin virtual substrates. Solid State Electron, 2004, 48: 1257-1263

6 Yamamoto Y, Kopke K, Weidner G, et al. Epitaxial growth of $\mathrm{Si}$ C/Si/SiGe into cavity formed by selective etching of SiGe. Solid State Electron, 2009, 53: 824-827

7 Jang J H, Phen M S, Siebein K, et al. Observation of defects evolution in strained SiGe layers during strain relaxation. Mater Lett, 2009, 
63: 289-291

8 Kamins T I, Meyer D J. Kinetics of silicon-germanium deposition by atmospheric-pressure chemical vapor-deposition. Appl Phys Lett, 1991, 59: 178-180

9 Deboer W B, Meyer D J. Low-temperature chemical vapor-deposition of epitaxial $\mathrm{Si}$ and $\mathrm{SiGe}$ layers at atmospheric-pressure. Appl Phys Lett, 1991, 58: 1286-1288

10 Di Z F, Zhang M, Liu W L, et al. Fabrication of thick, high-quality strained SiGe layer on ultra-thin silicon-on-insulator and modeling of film strain. Mat Sci Semicon Proc, 2004, 7: 393-397

11 Menon C, Radamson H H, Landgren G. High quality SiGe epitaxial layer grown by RPCVD using Dichlorosilane. The Third International Euro Conference on Advanced Semiconductor Devices and Microsystems, 2000 Oct. 16-18, Slovakia. 201-204

12 Hartmann J M, Loup V, Rolland G, et al. SiGe growth kinetics and doping in reduced pressure-chemical vapor deposition. J Cryst Growth, 2002, 236: 10-20

13 Hartmann J M, Loup V, Rolland G, et al. Effects of temperature and $\mathrm{HCl}$ flow on the SiGe growth kinetics in reduced pressure chemical vapor deposition. J Vacuum Sci Tech B: Microelect Nanometer Struct, 2003, 21: 2524-2529

14 Hartmann J M, Champay F, Loup V, et al. Effect of $\mathrm{HCl}$ on the SiGe growth kinetics in reduced pressure-chemical vapor deposition. J Cryst Growth, 2002, 241: 93-100

15 Hartmann J M, Papon A M, Barnes J P, et al. Growth kinetics of Si$\mathrm{Ge} / \mathrm{Si}$ superlattices on bulk and silicon-on-insulator substrates for multi-channel devices. J Cryst Growth, 2009, 311: 3152-3157

16 Holy V, Pietsch U, Baumbach T. High Resolution X-Ray Scattering from Thin Films and Multilayers, Springer Tracts in Modern Physics, Vol.149. Heidelberg: Springer, 1999

17 Hartmann J M, Bertin F, Rolland G, et al. Effects of the temperature and of the amount of $\mathrm{Ge}$ on the morphology of Ge islands grown by reduced pressure-chemical vapor deposition. Thin Solid Films, 2005, 479: 113-120

18 Hierlemann M, Werner C. Modeling of SiGe deposition using quantum chemistry techniques for detailed kinetic analysis. Mat Sci Semicon Proc, 2000, 3: 31-39

19 Cheng C L, Tsai D S, Jiang J C. Study of pathway of hydrogen migration and desorption on $\mathrm{SiGe}(100)$ surface using ab initio calculations. Jpn J Appl Phys, 2005, 44: 7625

20 Zhou Z, Li C, Lai H, et al. The influence of low-temperature Ge seed layer on growth of high-quality $\mathrm{Ge}$ epilayer on $\mathrm{Si}\left(\begin{array}{lll}1 & 0 & 0\end{array}\right)$ by ultrahigh vacuum chemical vapor deposition. J Cryst Growth, 2008, 310: 2508-2513

21 Suh K Y, Lee $\mathrm{H}$ H. Ge composition in $\mathrm{Si}_{1-x} \mathrm{Ge}_{x}$ films grown from $\mathrm{SiH}_{2} \mathrm{Cl}_{2} / \mathrm{GeH}_{4}$ precursors. J Appl Phys, 2000, 88: 4044-4047

22 Tsang J C, Mooney P M, Dacol F, et al. Measurements of alloy composition and strain in thin $\mathrm{Ge}_{\mathrm{x}} \mathrm{Si}_{1-x}$ layers. J Appl Phys, 1994, 75 : 8098-8108

23 Maree P, Barbour J, Veen J, et al. Generation of misfit dislocations in semiconductors. J Appl Phys, 1987, 62: 4413-4420

24 Kasper E, Lyutovich K, Bauer M, et al. New virtual substrate concept for vertical MOS transistors. Thin Solid Films, 1998, 336: 319-322

25 Lutz M A, Feenstra R M, Legoues F K, et al. Influence of misfit dislocations on the surface morphology of $\mathrm{Si}_{1-x} \mathrm{Ge}_{x}$ films. Appl Phys Lett, 1995, 66: 724-726

Open Access This article is distributed under the terms of the Creative Commons Attribution License which permits any use, distribution, and reproduction in any medium, provided the original author(s) and source are credited. 